\title{
Experimental Determination of Ramsey Numbers
}

\author{
Zhengbing Bian, ${ }^{1}$ Fabian Chudak, ${ }^{1}$ William G. Macready, ${ }^{1}$ Lane Clark, ${ }^{2}$ and Frank Gaitan ${ }^{3}$ \\ ${ }^{1}$ D-Wave Systems, Inc., 100-4401 Still Creek Drive, Burnaby, British Columbia V5C 6G9, Canada \\ ${ }^{2}$ Department of Mathematics, Southern Illinois University, Carbondale, Illinois 62901-4401, USA \\ ${ }^{3}$ Laboratory for Physical Sciences, 8050 Greenmead Drive, College Park, Maryland 20740, USA \\ (Received 2 June 2012; revised manuscript received 31 July 2013; published 25 September 2013)
}

\begin{abstract}
Ramsey theory is a highly active research area in mathematics that studies the emergence of order in large disordered structures. Ramsey numbers mark the threshold at which order first appears and are extremely difficult to calculate due to their explosive rate of growth. Recently, an algorithm that can be implemented using adiabatic quantum evolution has been proposed that calculates the two-color Ramsey numbers $R(m, n)$. Here we present results of an experimental implementation of this algorithm and show that it correctly determines the Ramsey numbers $R(3,3)$ and $R(m, 2)$ for $4 \leq m \leq 8$. The $R(8,2)$ computation used 84 qubits of which 28 were computational qubits. This computation is the largest experimental implementation of a scientifically meaningful adiabatic evolution algorithm that has been done to date.
\end{abstract}

DOI: 10.1103/PhysRevLett.111.130505

PACS numbers: 03.67.Ac, 02.10.Ox, 89.75.Hc

In recent years first steps have been taken towards experimentally realizing the computational advantages promised by well-known quantum algorithms. As with any nascent effort, these initial steps have been limited. To date the largest experimental implementations of scientifically meaningful quantum algorithms have used just a handful of qubits. For circuit-based algorithms [1], seven spin qubits were used to factor 15 , while for adiabatic algorithms [2], four spin qubits were used to factor 143. In both cases compiled versions of the algorithms were needed to allow factoring with such small numbers of qubits. Although factoring was the focus of both experiments, other scientifically significant applications exist.

In Ref. [3] an algorithm for determining the two-color Ramsey numbers was proposed which could be implemented using adiabatic quantum evolution. Ramsey numbers are part of an active research area in mathematics known as Ramsey theory [4] whose central theme is the emergence of order in large disordered structures. The disordered structures can be represented by an $N$-vertex graph $G$, and the ordered substructures by specific graphs $H_{1}$ and $H_{2}$ that are to appear as subgraphs of $G$. For two-color Ramsey numbers the subgraphs $H_{1}$ and $H_{2}$ are $m$-cliques and $n$-independent sets, respectively. An $m$-clique is a set of $m$ vertices that has an edge connecting any two of the $m$ vertices, and an $n$-independent set is a set of $n$ vertices in which no two of the $n$ vertices are joined by an edge. Using Ramsey theory [4,5], one can prove that a threshold value $R(m, n)$ exists so that for $N \geq R(m, n)$ every graph with $N$ vertices will contain either an $m$-clique or an $n$-independent set. The threshold value $R(m, n)$ is an example of a two-color Ramsey number. Other types of Ramsey numbers exist, though we focus on two-color Ramsey numbers here. Ramsey numbers $R(m, n)$ grow extremely quickly and are notoriously difficult to calculate. In fact, for $m, n \geq 3$, only nine are presently known [5].

In the Ramsey number algorithm (RNA) [3], the calculation of $R(m, n)$ is formulated as an optimization problem which can be solved using adiabatic quantum evolution [6]. Here we present evidence of an experimental implementation of the RNA using adiabatic quantum evolution and show that it correctly determines the Ramsey numbers $R(3,3)$ and $R(m, 2)$ for $4 \leq m \leq 8$. The experimental computation of $R(8,2)$ used a total of 84 qubits of which 28 were computational qubits and applied an effective interaction coupling 28 qubits. To the best of our knowledge, this is the largest experimental implementation of a scientifically meaningful adiabatic evolution algorithm.

We begin this Letter with a brief description of the RNA. We then discuss the details of experimentally implementing the RNA using adiabatic quantum evolution on a chip of 106 superconducting flux qubits, and follow with a presentation of our experimental results. Finally, we close with a discussion of what has been found.

I. Ramsey number algorithm.-We briefly describe the construction of the RNA; see Ref. [3] and the Supplemental Material [7] for a detailed presentation.

As computation of $R(m, n)$ is intimately connected with the presence or absence of edges, we associate a bit variable $a_{v, v^{\prime}}$ with each pair of vertices $\left(v, v^{\prime}\right)$ in an $N$-vertex graph $G$, and set $a_{v, v^{\prime}}=1(0)$ when $v$ and $v^{\prime}$ are (are not) joined by an edge in $G$. There are thus $L_{N}=\left(\begin{array}{c}N \\ 2\end{array}\right) \equiv N(N-1) / 2$ bit variables which we collect into the bit vector (bit string) $\boldsymbol{a}=\left(a_{2,1}, \ldots, a_{N, 1}, a_{3,2}, \ldots, a_{N, 2}, \ldots, a_{N, N-1}\right)$ of length $L_{N}$. Thus an $N$-vertex graph $G$ determines a unique bit string $\boldsymbol{a}$, and vice versa. Reference [3] (and the Supplemental Material [7]) showed how to count the number of $m$-cliques $C_{m}^{N}(\boldsymbol{a})$ and $n$-independent sets $I_{n}^{N}(\boldsymbol{a})$ in an $N$-vertex graph $G$ using its associated bit string $\boldsymbol{a}$. We can 
thus calculate the total number of $m$-cliques and $n$-independent sets contained in $G: h_{m, n}^{N}(\boldsymbol{a})=C_{m}^{N}(\boldsymbol{a})+$ $I_{n}^{N}(\boldsymbol{a})$. Now consider the following combinatorial optimization problem (COP): For given integers $(N, m, n)$ and cost function $h_{m, n}^{N}(\boldsymbol{a})$ defined as above, find an $N$-vertex graph $G_{a_{\star}}$ that yields the global minimum of $h_{m, n}^{N}(\boldsymbol{a})$. Notice that if $N<R(m, n)$, the global minimum is $h_{m, n}^{N}\left(\boldsymbol{a}_{\star}\right)=0$, since Ramsey theory guarantees that a graph exists that has no $m$-clique or $n$-independent set. Furthermore, if $N \geq$ $R(m, n)$, Ramsey theory guarantees that $h_{m, n}^{N}\left(\boldsymbol{a}_{\star}\right)>0$.

Adiabatic quantum optimization (AQO) [6] is a $T=0$ ground-state (GS) method that exploits the adiabatic evolution of a quantum system to solve COPs, while quantum annealing (QA) [8] is a finite temperature method which can also be used to solve COPs even in the presence of decoherence. Reference [3] described a quantum implementation of the RNA using AQO, while in this Letter we present evidence for a QA implementation of the RNA. (Note that a classical implementation of the RNA is also possible using a classical optimization algorithm run on a classical computer to solve the Ramsey number COP.) Both AQO and QA use the COP cost function to define a problem Hamiltonian $H_{P}$ whose ground-state eigenspace contains all COP solutions. These algorithms evolve the state of a qubit register from the ground state of an initial Hamiltonian $H_{i}$ to a ground state of $H_{P}$ with high probability in the adiabatic limit. The algorithm dynamics is driven by a time-dependent Hamiltonian $H(t)=$ $A\left(t / t_{f}\right) H_{i}+B\left(t / t_{f}\right) H_{P}$, where $t_{f}$ is the algorithm run time, adiabatic dynamics corresponds to $t_{f} \rightarrow \infty$, and $A\left(t / t_{f}\right)\left[B\left(t / t_{f}\right)\right]$ is a positive monotonically decreasing [increasing] function with $A(1)=0[B(0)=0]$.

In the quantum implementation of the RNA each bit variable $a_{v, v^{\prime}}$ is promoted to a qubit, thus associating a qubit with each vertex pair $\left(v, v^{\prime}\right)$. The bit strings $a$ now label the computational basis (CB) states $|\boldsymbol{a}\rangle$, and the problem Hamiltonian $H_{P}$ is defined to be diagonal in the CB with eigenvalue $h_{m, n}^{N}(\boldsymbol{a})$. By construction, the ground-state energy of $H_{P}$ vanishes if and only if there is a graph with no $m$-cliques or $n$-independent sets. The initial Hamiltonian $H_{i}$ is the standard one for AQO [6] and appears in the Supplemental Material [7]. Its unique ground state is the uniform superposition of all CB states.

Implementation of the RNA using QA computes $R(m, n)$ as follows. First, choose $N$ such that $N<R(m, n)$, then run QA on the $L_{N}$ qubits and measure the qubits in the CB at the end of the anneal. This yields a bit string $\boldsymbol{a}_{*}$, which determines the final energy $E=h_{m, n}^{N}\left(\boldsymbol{a}_{*}\right)$. In the adiabatic limit the result will be $E=0$ since $N<R(m, n)$. Now increment $N \rightarrow N+1$, rerun QA on the $L_{N+1}$ qubits, and measure the final energy. Repeatedly increment $N$ until $E>0$ first occurs, at which point the current value of $N$ will be equal to $R(m, n)$. In any real application of the above algorithm the evolution will only be approximately adiabatic, and the probability that the measured energy will be the ground-state energy will thus be $1-\epsilon$. By running the algorithm $k \sim O(\ln [1-\delta] / \ln \epsilon)$ times, the probability $\delta$ that at least one of the measurement outcomes yields the ground-state energy can be made arbitrarily close to 1 .

II. Experimental implementation.-Our hardware is designed to implement QA using rf SQUID flux qubits. Each qubit is a superconducting loop interrupted by Josephson junctions, and the states $|0\rangle$ and $|1\rangle$ correspond to the two directions of circulating current about the loop [9]. The chip hardware uses Josephson-junction-based devices to produce pairwise qubit coupling [10]. By rescaling the chip Hamiltonian by the interqubit coupling energy $J_{\mathrm{AFM}}(t)=M_{\mathrm{AFM}}\left|I_{q}^{p}(t)\right|^{2}$, the low-energy dynamics of the chip can be represented by a quantum Ising model in a transverse field with the Hamiltonian [11],

$$
H=-A\left(\frac{t}{t_{f}}\right) \sum_{i} \sigma_{i}^{x}+B\left(\frac{t}{t_{f}}\right)\left\{\sum_{i} h_{i} \sigma_{i}^{z}+\sum_{(i, j) \in E} J_{i j} \sigma_{i}^{z} \sigma_{j}^{z}\right\}
$$

Here $M_{\mathrm{AFM}}$ is the maximum antiferromagnetic (AFM) value of the interqubit effective mutual inductance that the hardware can produce, and $I_{q}^{p}(t)$ is the supercurrent circulating about the rf SQUID loop. Although the scale factor $J_{\mathrm{AFM}}(t)$ is time dependent, its order of magnitude can be estimated. For the D-Wave One device, $M_{\mathrm{AFM}}=$ $1.8 \mathrm{pH}$, and $\left|I_{q}^{p}(t)\right| \sim 0.6 \mu \mathrm{A}$ at the quantum critical point, so that $J_{\mathrm{AFM}} \sim 1 \mathrm{GHz}$ or $50 \mathrm{mK}$. With this rescaling, the local biases $\left\{h_{i}\right\}$ and coupling strengths $\left\{J_{i j}\right\}$ may be programed to values in the ranges $[-2,2]$ and $[-1,1]$, respectively, and the experimentally measured functional forms of the interpolation functions $A\left(t / t_{f}\right)$ and $B\left(t / t_{f}\right)$ appear in the Supplemental Material [7], along with the layout of qubits and couplers on the chip. For further details of the chip hardware, see Ref. [12].

The cost function $h_{m, n}^{N}(\boldsymbol{a})$ is not yet ready for experimental implementation for two reasons: (a) there are $k$-qubit interactions with $k>2$ and (b) the qubit couplings do not correspond to the qubit couplings on the chip. These obstacles are removed as follows.

(a) Reduction to pairwise coupling: The Supplemental Material [7] shows that $C_{m}^{N}(\boldsymbol{a})$ involves interactions coupling $\left(\begin{array}{c}m \\ 2\end{array}\right)$ qubits, while $I_{n}^{N}(\boldsymbol{a})$ couples $\left(\begin{array}{c}n \\ 2\end{array}\right)$ qubits. These interactions must be reduced to pairwise coupling if $h_{m, n}^{N}(\boldsymbol{a})$ is to be realized experimentally. We illustrate how such a reduction can be achieved by reducing the 3 -bit coupling term $a_{1} a_{2} a_{3}$ to pairwise coupling using an ancillary bit variable $b$ and penalty function $P\left(a_{1}, a_{2} ; b\right)=$ $a_{1} a_{2}-2\left(a_{1}+a_{2}\right) b+3 b$. Notice that $P\left(a_{1}, a_{2} ; b\right)=0(>0)$ when the input values for $a_{1}, a_{2}$, and $b$ satisfy $b=a_{1} a_{2}\left(b \neq a_{1} a_{2}\right)$. Now consider the quadratic cost function $h(b)=b a_{3}+\mu P\left(a_{1}, a_{2} ; b\right)$ for given values of $\mu$ and $a_{i}$. For $\mu$ sufficiently large, $h(b)$ is minimized when $b$ satisfies the equality constraint $b=a_{1} a_{2}$ which causes the penalty function to vanish. The optimum cost is then $h\left(b=a_{1} a_{2}\right)=a_{1} a_{2} a_{3}$ which reproduces the 3-bit 
coupling term using a quadratic cost function. This example is generalized in the Supplemental Material [7] to produce the quadratic cost function used to calculate $R(m, 2)$.

(b) Matching spin to qubit connectivity: A cost function with only pairwise qubit coupling may still not be experimentally realizable as the qubit couplings needed may not match the qubit couplings available on chip. The primal graph (PG) of a quadratic cost function is the graph whose vertices are the qubit variables, and whose edges indicate pairwise-coupled qubits. An arbitrary PG can be embedded into a sufficiently large qubit graph having the qubit layout and connectivity present on the chip. An embedding maps a PG vertex to one or more vertices in the qubit graph, where the image vertices form a connected subgraph of the qubit graph. We link this connected set of qubits together with strong ferromagnetic couplings of strength $\lambda$ so that in the lowest energy state these qubits have identical Bloch vectors. An example of this ferromagnetic coupling procedure is given in the Supplemental Material [7].

III. Embedding Ramsey problems.-We examined all Ramsey number COPs that could be solved using the 106 qubits available on the chip. Specifically, $R(m, 2)$ with $4 \leq m \leq 8$, and $R(3,3)$. Here we describe the embedding of these problems onto the chip.

(a) $R(m, 2)$ : Since an $N$-vertex graph $G_{\boldsymbol{a}}$ with $N<m$ cannot contain an $m$-clique, it follows that $C_{m}^{N}(\boldsymbol{a})=0$ for all such $G_{\boldsymbol{a}}$. Thus, for $N<m, h_{m, 2}^{N}(\boldsymbol{a})=I_{2}^{N}(\boldsymbol{a})=\bar{a}_{1}+$ $\cdots+\bar{a}_{L_{N}}$, where $\bar{a}_{i}=1-a_{i}$. This produces a problem Hamiltonian $H_{P}$ with $L_{N}$ uncoupled qubits, which is easily mapped onto the chip. Now consider $N=m$. Defining $L=L_{m}=\left(\begin{array}{c}m \\ 2\end{array}\right)$, we have $C_{m}^{m}(\boldsymbol{a})=a_{1} a_{2} \cdots a_{L-1} a_{L}$, and $h_{m, 2}^{m}(\boldsymbol{a})=C_{m}^{m}(\boldsymbol{a})+I_{2}^{m}(\boldsymbol{a})$. The $L$-bit interaction in $C_{m}^{m}(\boldsymbol{a})$ is reduced to pairwise coupling by (i) introducing ancillary bit variables $b_{2}, \ldots, b_{L-1}$ and (ii) imposing the constraints $b_{L-1}=a_{L-1} a_{L} \quad$ and $\quad b_{j}=a_{j} b_{j+1} \quad(j=2, \ldots, L-2)$ through the penalty function $P(\boldsymbol{a} ; \boldsymbol{b})=P\left(a_{L-1}, a_{L}\right.$; $\left.b_{L-1}\right)+\sum_{j=2}^{L-2} P\left(a_{j}, b_{j+1} ; b_{j}\right)$, where $P(a, b ; c)$ was defined in Sec. II(a). The $R(m, 2)$ cost function for $N=m$ is then $h_{m, 2}^{m}(\boldsymbol{a}, \boldsymbol{b})=\left\{a_{1} b_{2}+\mu P(\boldsymbol{a} ; \boldsymbol{b})\right\}+I_{2}^{m}(\boldsymbol{a})$, where $\mu=2$ is the penalty weight value used in all $R(m, 2)$ experiments. Making the substitutions $2 \boldsymbol{a}=$ $\boldsymbol{s}_{a}+1$ and $2 \boldsymbol{b}=\boldsymbol{s}_{b}+1$ expresses the cost function in terms of Ising spin variables $\boldsymbol{s}_{a}$ and $\boldsymbol{s}_{b}$. The PG for the pairwise interactions present in $h_{m, 2}^{m}(\boldsymbol{a}, \boldsymbol{b})$ appears in the Supplemental Material [7]. We have embedded this PG into the hardware up to $N=m=8$. In [7] we display the embedding that was used to determine $R(8,2)$ which used 28 computational qubits, 26 ancilla qubits to reduce interactions to pairwise, and 30 qubits to match the PG connectivity to the qubit connectivity available on the chip for a total of 84 qubits.

(b) $R(3,3)$ : We also determined $R(3,3)$ by examining $N=4,5,6$. The cost functions for these cases are

$$
\begin{aligned}
h_{3,3}^{4}(\boldsymbol{a})= & f_{1,2,4}+f_{1,3,5}+f_{2,3,6}+f_{4,5,6}, \\
h_{3,3}^{5}(\boldsymbol{a})= & f_{1,2,5}+f_{1,3,6}+f_{1,4,7}+f_{2,3,8}+f_{2,4,9}+f_{3,4,10} \\
& +f_{5,6,8}+f_{5,7,9}+f_{6,7,10}+f_{8,9,10}, \\
h_{3,3}^{6}(\boldsymbol{a})= & f_{1,2,6}+f_{1,3,7}+f_{1,4,8}+f_{1,5,9}+f_{2,3,10}+f_{2,4,11} \\
& +f_{2,5,12}+f_{3,4,13}+f_{3,5,14}+f_{4,5,15}+f_{6,7,10} \\
& +f_{6,8,11}+f_{6,9,12}+f_{7,8,13}+f_{7,9,14}+f_{8,9,15} \\
& +f_{10,11,13}+f_{10,12,14}+f_{11,12,15}+f_{13,14,15},
\end{aligned}
$$

where $f_{i, j, k}=a_{i} a_{j} a_{k}+\bar{a}_{i} \bar{a}_{j} \bar{a}_{k}$. Notice that $f_{i, j, k}$ can be rewritten as $f_{i, j, k}=-2+\bar{a}_{i}+\bar{a}_{j}+\bar{a}_{k}+a_{i} a_{j}+a_{i} a_{k}+$ $a_{j} a_{k}$, which only contains pairwise couplings, making ancillary $\boldsymbol{b}$ qubits unnecessary. The largest of these problems is for $N=6$ whose PG has 15 vertices and 60 edges. We can reduce its size slightly by exploiting the identity $h_{m, n}^{N}(\boldsymbol{a})=h_{n, m}^{N}(\overline{\boldsymbol{a}})$. For $m=n$ this yields a twofold symmetry: if $\boldsymbol{a}_{\star}$ is a global minimum of $h_{m, m}^{N}$, so is $\overline{\boldsymbol{a}}_{\star}$. Thus, we can fix one variable (say, $a_{1}=0$ ) and optimize over the remaining variables $\boldsymbol{a}^{\prime}$. Optimal solutions then have the form $\left(0, \boldsymbol{a}_{\star}^{\prime}\right)$ and $\left(1, \overline{\boldsymbol{a}}_{\star}^{\prime}\right)$. With this simplification, the PG of $h_{3,3}^{6}\left(0, \boldsymbol{a}^{\prime}\right)$ has 14 vertices and 52 edges. We show its embedding into the chip hardware in the Supplemental Material [7].

IV. Results.-To solve a given Ramsey COP specified by the parameters $\boldsymbol{h}$ and $\boldsymbol{J}$, the chip must first be programed to fix these values in the hardware. For the largest problem we solved $[R(8,2)$ using 84 qubits], this took roughly $270 \mathrm{~ms}$. After programing we iterate many cycles of annealing and readout. Each annealing cycle has duration $t_{f}=1 \mathrm{~ms}$, and readout of the qubits takes $1.5 \mathrm{~ms}$ per sample. Programing only occurs once so the total run time required to obtain $S$ Ramsey output samples is $270+(1+1.5) S \mathrm{~ms}$. As the hardware is an analog device, there is limited precision to which $\boldsymbol{h}$ and $\boldsymbol{J}$ can be specified. For a COP whose ground state is sensitive to parameter settings, this could pose serious difficulties. However, the Ramsey COP requires specification of only a few distinct integral values, and ground states are quite stable to parameter perturbations. The reader is referred to the Supplemental Material [7] for further discussion of (i) the quantum annealing rate and the distribution of final energies, (ii) the experimental temperature, and (iii) parameter noise.

Figures 1 and 3 present our results for $R(8,2)$ and $R(3,3)$. Because of space limitations, our full set of results $[R(3,3)$ and $R(m, 2)$ with $4 \leq m \leq 8]$ appears in the Supplemental Material [7], though Table I contains a summary of all results, along with corresponding theoretical predictions. Both Figs. 1 and 3 display histograms that plot the relative frequency of energy values obtained by programing the chip and running $10^{5}$ annealing and readout cycles, yielding $10^{5} \mathrm{~s}$-spin configurations. In the main figures, histograms of the energies of the Ising problem sent to the hardware are plotted. These Ising cost functions 


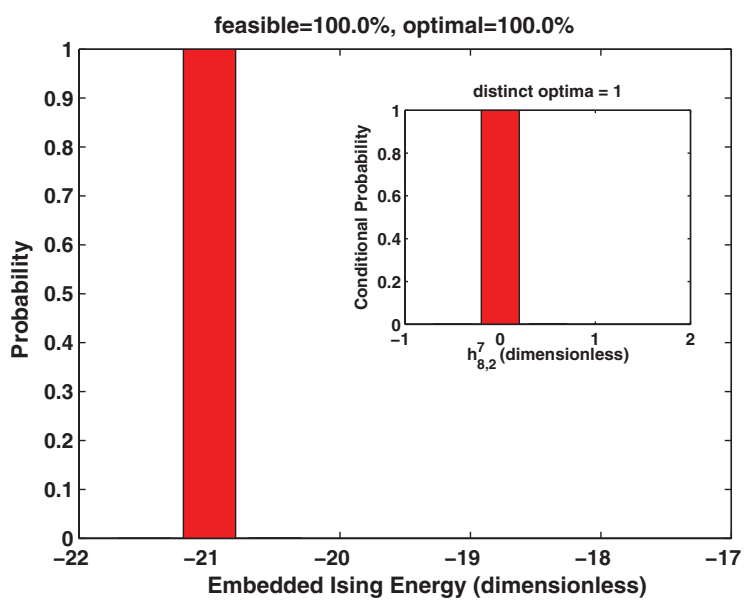

(a)

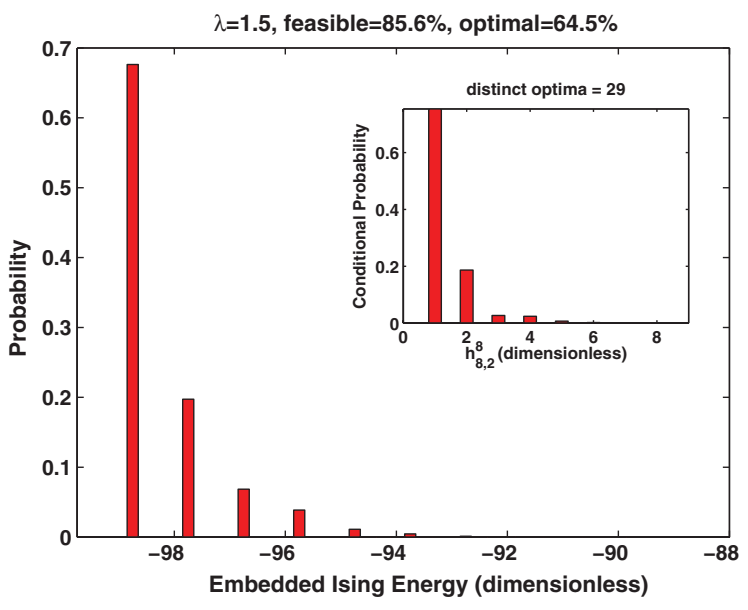

(b)

FIG. 1 (color). Energy histograms for $R(8,2)$ for graphs with: (a) $N=7$ and (b) $N=8$, which are, respectively, below and at the Ramsey threshold. Inset: histogram of relative frequency of feasible $\boldsymbol{a}$ configurations vs Ramsey energy $h_{8,2}^{N} N(\boldsymbol{a})$.

include the Ramsey cost function $h_{m, n}^{N}(s)$ and the ferromagnetic penalties $\lambda$, enforcing the equality constraints among qubits that represent the same PG spin variable. The ferromagnetic penalty weight used for embedding was adjusted so that at least $85 \%$ of output $s$ configurations satisfied the equality constraints. These feasible spin configurations were translated back to the original $\boldsymbol{a}$ variables and the cost-energy function $h_{m, n}^{N}(\boldsymbol{a})$ evaluated. The

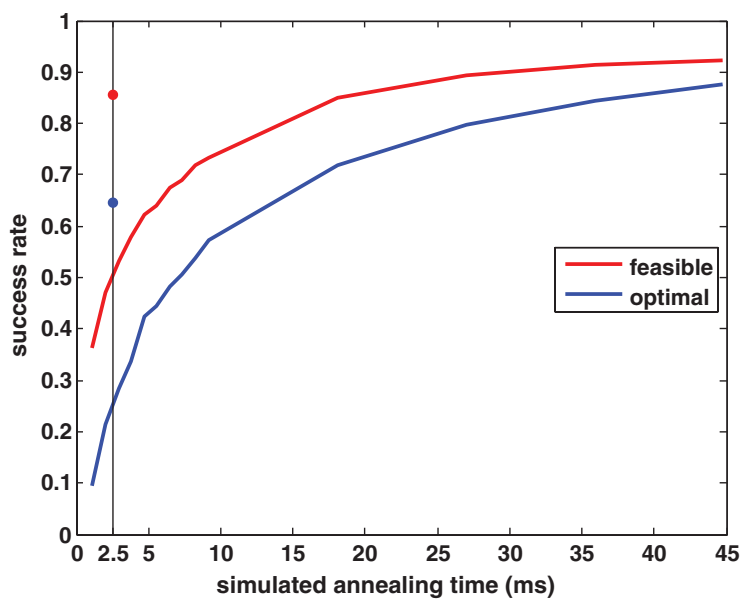

FIG. 2 (color). Plot of simulated annealing success probability to determine optimal and feasible spin configurations versus run time for $R(8,2)$ at $N=8$. The simulated annealing cooling schedule is exponential, and the initial and final temperatures were optimized for maximum success probability. For comparison, the D-Wave hardware results are also shown for a run time of $2.5 \mathrm{~ms}$. See text for further discussion.

resulting energy values were binned and plotted in the inset histograms. Further discussion of the equality constraint protocol appears in the Supplemental Material [7].

Figure 1 presents our results for $R(8,2)$ for $N=7$ and 8 , while $N=6$ appears in [7]. The $R(8,2)$ experiment was the largest of the $R(m, 2)$ problems considered. Of the $s$ configurations returned by the hardware for $N=8$, approximately $65 \%$ are global minima of $h_{8,2}^{8}$. By comparison, steepest-descent local search started from a random spin configuration finds a globally minimal configuration with less than $0.01 \%$ probability. Note that classical or thermal annealing can be ruled out as the source of optimization efficacy. First, it is clear that the hardware is not realizing classical annealing since the final distribution of low-energy states is not Boltzmann distributed as discussed in the Supplemental Material [7], Sec. VIB, and furthermore, the temperature of the hardware is never varied during the experiments. Finally, we compare the optimization efficacy of the hardware with that of an efficient $\mathrm{C}$ implementation of simulated annealing that was run on a standard $8 \mathrm{~Gb}, 2.66 \mathrm{GHz}$ desktop computer. The results of Fig. 2 show that, at a run time of $2.5 \mathrm{~ms}$ (which is the $1 \mathrm{~ms}$ run time plus 1.5 ms readout time of the Ramsey number

TABLE I. Results for Ramsey numbers $R(m, 2)=m$ for $4 \leq m \leq 8$ and $R(3,3)=6$. Here $N$ is the number of graph vertices; $E_{\mathrm{GS}}$ and $D$ are the ground-state energy and degeneracy, respectively, for the problem Hamiltonian $H_{P}$; and for each Ramsey number, the experimental results are followed by the theoretical predictions from Ref. [3] in parenthesis.

\begin{tabular}{lcccccccccccccccccc}
\hline \hline \multicolumn{2}{ll}{$R(2,4)$} & \multicolumn{1}{l}{$R(2,5)$} & \multicolumn{4}{c}{$R(2,6)$} & \multicolumn{1}{c}{$R(2,7)$} & \multicolumn{3}{c}{$R(2,8)$} & \multicolumn{4}{c}{$R(3,3)$} \\
\hline$N$ & $E_{\mathrm{GS}}$ & $D$ & $N$ & $E_{\mathrm{GS}}$ & $D$ & $N$ & $E_{\mathrm{GS}}$ & $D$ & $N$ & $E_{\mathrm{GS}}$ & $D$ & $N$ & $E_{\mathrm{GS}}$ & $D$ & $N$ & $E_{\mathrm{GS}}$ & $D$ \\
\hline 3 & $0(0)$ & $1(1)$ & 4 & $0(0)$ & $1(1)$ & 5 & $0(0)$ & $1(1)$ & 6 & $0(0)$ & $1(1)$ & 7 & $0(0)$ & $1(1)$ & 5 & $0(0)$ & $12(12)$ \\
4 & $1(1)$ & $7(7)$ & 5 & $1(1)$ & $11(11)$ & 6 & $1(1)$ & $16(16)$ & 7 & $1(1)$ & $22(22)$ & 8 & $1(1)$ & $29(29)$ & 6 & $2(2)$ & $1758(1760)$ \\
\hline \hline
\end{tabular}




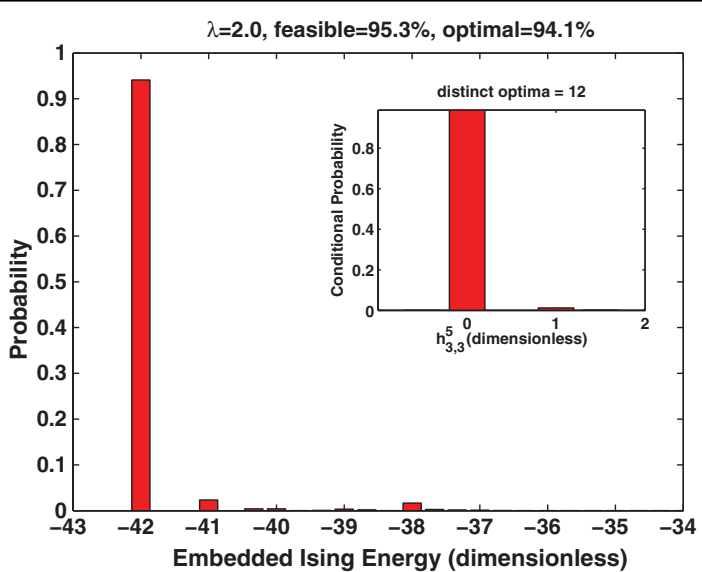

(a)

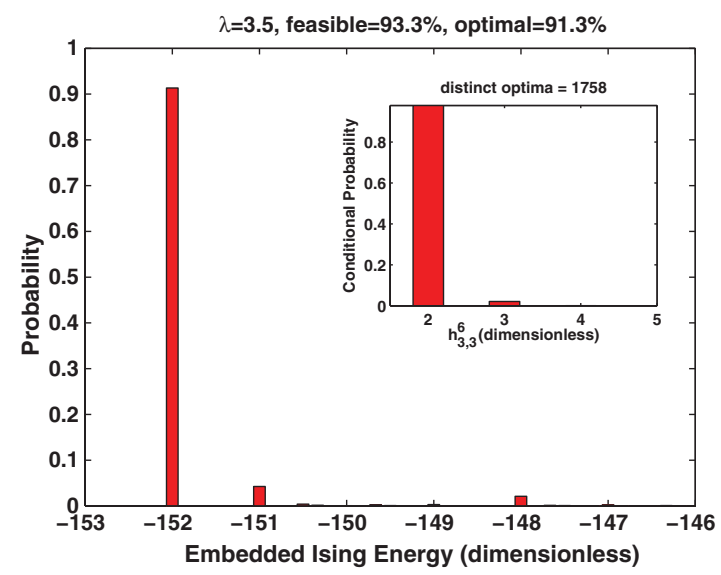

(b)

FIG. 3 (color). Energy histograms for $R(3,3)$ for graphs with: (a) $N=5$ and (b) $N=6$ which are, respectively, below and at the Ramsey threshold. Inset: histogram of relative frequency of feasible $\boldsymbol{a}$ configurations vs Ramsey energy $h_{3,3}^{N}(\boldsymbol{a})$.

experiments), the hardware obtains significantly higher success rates for finding both feasible and optimal final spin configurations than does simulated annealing. Returning to Fig. 1, examination of the inset histograms for $N=7$ (8), we see that (i) $h_{\min }=0(1)$, (ii) the probability for $h=0(1)$ is approximately 1.0(0.65), and (iii) the number of optimal $\boldsymbol{a}$ configurations or graphs is 1 (29). The reader is referred to the Supplemental Material [7] for an explanation of how the probability for an optimal spin configuration is determined. The energies $h_{\min }$ found for $N=7$ and 8 agree with the final GS energies found in Ref. [3], indicating that the hardware finds the final GS with high probability. As $h_{\min }$ jumps from $0 \rightarrow 1$ as $N$ goes from $7 \rightarrow 8$, the Ramsey protocol correctly [5] identifies $R(8,2)=8$. Finally, Ref. [3] showed that the number of optimal graphs for $N=7(8)$ is 1 (29), which agrees with what was found by the hardware. For $N=7$, the unique optimal $\boldsymbol{a}$ configuration corresponds to the graph in which every pair of vertices is connected by an edge and so has no 2-independent sets or 8 -clique and so has $h_{8,2}^{7}=0$. For $N=8$, the 29 optimal $\boldsymbol{a}$ configurations correspond to graphs $G_{\boldsymbol{a}}$ with $h_{8,2}^{8}(\boldsymbol{a})=1$, which are the 28 eight-vertex graphs containing a single 2-independent set, and the single 8-vertex graph containing an 8-clique.

Figure 3 shows our results for $R(3,3)$ with $N=5$ and 6 , while $N=4$ appears in the Supplemental Material [7]. Together, they show that the minimum energies for $N=4,5,6$ are $0,0,2$, respectively, and these occur with probabilities of approximately $93 \%, 94 \%$, and $91 \%$. These success rates are much higher than steepest-descent local search. The success rate for randomly initialized local search is less than $1 \%$ for $N=6$. The energies $h_{\min }$ agree with the final GS energies found in Ref. [3], indicating that the hardware again finds the final GS with high probability. As $h_{\text {min }}$ jumps from $0 \rightarrow 2$ as $N$ goes from $5 \rightarrow 6$, the Ramsey protocol correctly [5] identifies $R(3,3)=6$. Finally, Ref. [3] showed that the number of optimal graphs for $N=4,5,6$ is $18,12,1760$, respectively, in excellent agreement with the hardware results of 18, 12, 1758.

V. Discussion.-We presented results of an experimental implementation of the RNA [3]. As the Ramsey numbers found correspond to known Ramsey numbers, it was possible to validate RNA performance. Agreement between theory and experiment was excellent: experimental implementation of the RNA correctly determined (i) $R(m, 2)=m$ for $4 \leq m \leq 8$ and $R(3,3)=6$ and (ii) the corresponding final ground-state energies and degeneracies. Our results provide evidence of a quantum implementation of the RNA based on adiabatic evolution. Further evidence that the D-Wave hardware implements quantum annealing has recently been reported [13]. It was argued in Ref. [14] that this evidence did not imply quantum annealing, though these arguments were refuted in Ref. [15]. Finally, we stress that the optimization formulations necessary for experimental realization of the RNA were nontrivial. The Ising problems after embedding, solved with high success rate by the hardware, have many local minima which were responsible for the low success rates of iterated local search. In spite of the many local minima, the hardware implementation of the RNA correctly determined all of the above Ramsey numbers. The $R(8,2)$ computation used 84 qubits of which 28 were computational qubits, and to the best of our knowledge is the largest experimental implementation of a scientifically meaningful adiabatic evolution algorithm.

We thank the D-Wave hardware team for providing this experimental platform, and M. Amin, R. Harris, T. Lanting, and M. Thom for valuable suggestions. F. G. thanks T. Howell III for continued support.

[1] L. M. K. Vandersypen, M. Steffen, G. Breyta, C. S. Yannoni, M. H. Sherwood, and I. L. Chuang, Nature (London) 414, 883 (2001). 
[2] N. Xu, J. Zhu, D. Lu, X. Zhou, X. Peng, and J. Du, Phys. Rev. Lett. 108, 130501 (2012).

[3] F. Gaitan and L. Clark, Phys. Rev. Lett. 108, 010501 (2012).

[4] R. L. Graham, B. L. Rothschild, and J. H. Spencer, Ramsey Theory (John Wiley \& Sons, New York, 1990).

[5] B. Bollobás, Modern Graph Theory (Springer, New York, 1998).

[6] E. Farhi, J. Goldstone, S. Gutmann, and M. Sipser, arXiv: quant-ph/0001106.

[7] See Supplemental Material at http://link.aps.org/ supplemental/10.1103/PhysRevLett.111.130505 for details of the algorithm, its experimental implementation, and all experimental results.
[8] T. Kadowaki and H. Nishimori, Phys. Rev. E 58, 5355 (1998); G. E. Santoro, R. Martoňák, E. Tosatti, and R. Car, Science 295, 2427 (2002).

[9] R. Harris et al., Phys. Rev. B 81, 134510 (2010).

[10] R. Harris, T. Lanting, A. J. Berkley, J. Johansson, M. W. Johnson, P. Bunyk, E. Ladizinsky, N. Ladizinsky, T. Oh, and S. Han, Phys. Rev. B 80, 052506 (2009).

[11] R. Harris et al., Phys. Rev. B 82, 024511 (2010).

[12] M. W. Johnson et al., Nature (London) 473, 194 (2011).

[13] S. Boixo, T. Albash, F. M. Spedalieri, N. Chancellor, and D. A. Lidar, Nat. Commun. 4, 2067 (2013).

[14] J. A. Smolin and G. Smith, arXiv:1305.4904.

[15] L. Wang et al., arXiv:1305.5837. 